\title{
Los valores sobre la participación de la infancia en la vida familiar: un análisis de la encuesta mundial de valores en Andalucía
}

Juan Miguel GÓMEZ ESPINO*

Rosalía MARTÍNEZ GARCÍA*

\section{RESUMEN}

En esta comunicación se trata de analizar en qué medida la transformación social operada en las sociedades contemporáneas puede estar afectando a la relación adultos-infancia en lo referente a los modelos de participación de los niños en la vida familiar.

Para el estudio empírico de esta cuestión hemos hecho uso de la base de datos de la Encuesta Mundial de Valores, Andalucía (1998), de la que se han seleccionado ítems especialmente útiles para el objetivo de esta investigación, esto es, aquéllos que nos informan sobre las concepciones de los adultos respecto a la educación de los niños en el contexto de las relaciones de familia.

\section{PALABRAS CLAVE}

Infancia, participación, valores, cambio familiar, socialización, autonomía, heteronomía. 


\section{ABSTRACT}

In this paper, we pretend to analyze how and in what extent the social change in contemporary societies, over all in the relationship among adults and childhood, has involved more prominence of the children in a sense of more participation level in the familiar life.

For the empirical research, it will be used the data base of the World Survey of Values, in particular, the results related to Andalusia, obtained in 1998. From this survey, it has been selected various items that report about the adults conceptions in relations to the childhood, especially in the context of the family relationship.

Key words: childhood, participation, values, family change, socialization, autonomy, heteronomy.

\section{INFANCIA Y MODERNIZACIÓN}

La etapa infantil de la vida de los individuos ha estado con frecuencia excluida del análisis sociológico. De alguna manera, se consideraba a la infancia como un espacio "presocial" en el que los niños se transformaban progresivamente en sujetos plenos, lo que coincidía con la llegada de la adultez. Aquélla se vinculaba más a un hecho biológico de tránsito a la madurez que a una realidad específicamente social, propiciándose una suerte de "naturalización" de la infancia en los acercamientos predominantes. En definitiva, se admitía más o menos expresamente que los niños se sometían a una suerte de "moratoria social" (Ferrán Casas, 1998) hasta convertirse en adultos.

Este acercamiento, que ha impregnado la investigación en la materia (y, en ocasiones, la ausencia de ésta), ha sido objeto de denuncias persistentes desde aproximaciones teóricas próximas a lo que se ha dado en llamar "(nueva) sociología de la Infancia". Desde este novedoso campo sociológico, se ha favorecido un novedoso tratamiento de la realidad de la infancia entre cuyas premisas se encuentra considerarla como "una forma particular y distinta de la estructura de cualquier sociedad" (Qvortrup, 1993), así como "agentes activos en la construcción de la vida social” (Brennen y O'Brien, 1996). En consecuencia, se ha considerado preciso definir 
la infancia antes por su interés intrínseco que por su carácter de fase transitoria hacia la adultez.

Desde la perspectiva del cambio social, este colectivo ha sido objeto de atención a través del análisis del proceso de modernización (y postmodernización) que ha sido emprendido en general con relación a nuestras sociedades contemporáneas y, en particular, a la institución familiar -vínculo fundamental entre los niños y la realidad social más amplia-. Los cambios producidos en la familia constituyen un factor explicativo fundamental de las transformaciones que se vislumbran en la concepción de la infancia, aunque bien es cierto que se deben extremar las cautelas para evitar caer en la "invisibilización" de su realidad a través de la adopción de un ángulo excesivamente "familiarista" (Qvortrup, 1992). En definitiva, entendemos que no es posible negar la evidencia de la enorme significación que tiene en la vida infantil la forma en que la familia procede a (re)construirse socialmente.

La familia ha sufrido transformaciones de enorme envergadura que han afectado de manera incuestionable a la misma concepción de la infancia. En este sentido, estaríamos de acuerdo con la afirmación de que los niños (especialmente en cuanto que "hijos") son mucho más importantes que en el pasado. Y con ello, no queremos eludir la sustancial paradoja que resulta de que, a pesar de esto, las cifras demográficas no parecen confirmar esta afirmación. En efecto, a pesar de que los hijos son más queridos cualitativamente (se tienen de forma voluntaria, no percibiéndose ya como consecuencia natural de la vida en matrimonio), cuantitativamente se ha reducido su número en nuestras sociedades occidentales. Según desde qué ángulo de la paradoja nos situemos, daremos luz de un modo u otro a lo que resulta una aparente antinomia. Para algunos, el hecho demográfico contradice la anterior afirmación, considerándose este factor como una fuente explicativa fundamental de la consideración (negativa) que estructuralmente recibe la infancia (Qvortrup, 1992). Para otros, en cambio, la mayor atención cualitativa de la infancia justifica por sí misma estrategias familiares dirigidas a disminuir su número y, por tanto, a maximizar los recursos en un número reducido de hijos (Gil Calvo, 2003; Alberdi, 1999; Flaquer, 1998). Esta situación se explicaría en un contexto de una nueva ética familiar en la que los hijos dejan de ser algo social y colectivo (una especie de obligación con la sociedad), convirtiéndose en una construcción esencialmente privada, resultado de las decisiones particulares y autónomas de las parejas. 
En este nuevo escenario caracterizado, además, por las posibilidades técnicas de control de la natalidad, tener hijos es el resultado de una decisión consciente y voluntaria que frecuentemente entra en contradicción con las demandas de libertad e independencia de los adultos. La idea de individualización del individuo postmoderno alude exactamente a esta tendencia según la cual el orden social más amplio pierde fuerza coercitiva frente a lo que ocurría en las sociedades del pasado. No se trata sólo de que los hijos hayan dejado de ser una fuente de producción (en el tiempo en que los niños venían, entonces sí, "con un pan debajo del brazo") para convertirse en factores de consumo, resultando, por eso mismo, costosos. Además, éstos han pasado a ser parte sustancial de un proyecto de vida en común que significa la pareja, como algo querido en sí mismo y no como mero desenlace natural de la vida familiar. Así, del control sobre las decisiones relativas a la natalidad y de los condicionantes de esa decisión, se deriva un determinado acercamiento sobre los frutos de la misma: los niños dejan de ser concebidos como producto consustancial de la institución familiar para pasar a ser entendidos como productos conscientes de los proyectos de cada familia. Como afirma Alberdi, "los hijos han entrado en el reino de la libertad y la voluntad y ya apenas son fruto del azar o de la necesidad" (Alberdi, 1999, pág. 156).

Este nuevo escenario ha suscitado la proliferación de un análisis fecundo en el ámbito de la "sociología de la infancia", resultando de especial interés el estudio del tránsito hacia la (post) modernización de la infancia a partir de las dimensiones de "individualización" e "institucionalización".

La "individualización" alude a un aspecto referente a la esfera de los valores. El proceso de "individualización" afecta a la propia conformación de la familia que se sustenta ahora antes en las decisiones autónomas de los adultos que en la necesidad de proyectar su existencia a partir de los dictados de la tradición, o, si se prefiere, de las presiones de la comunidad. La proliferación e importancia de una variedad de tipologías familiares (el llamado "pluralismo familiar") es el resultado lógico de su novedosa concepción. En la medida en que la "individualización" es un proceso que afecta a la vida social en general, los individuos se convierten en agentes movilizadores de sus propios destinos antes que en meros sujetos pasivos de los roles preasignados por un orden social estático. Desde la perspectiva de los niños, la "individualización" implica el reconocimiento de éstos como personalidades únicas, dignas, por consiguiente, de ser tratadas con específica atención y cuidado por quienes ostentan la responsabilidad de su adecuado desarrollo. 
Es evidente que la familia adquiere una posición fundamental a la hora de procurar los recursos necesarios (de tipo material y, cada vez más, afectivos), con lo que se pretende garantizar el éxito del proceso socializador, resultando éstos. Sin embargo, en este nuevo contexto, la socialización ya no se concibe como un proceso unidireccional dirigido a la asunción por parte de los niños de normas bien definidas en la vida social. También, las pautas "socializadoras" se modifican como resultado del reconocimiento de la autonomía de los niños a partir de sus rasgos de personalidad. Cada vez más, se valora que los niños sepan manejarse por sí mismos con la menor dependencia posible, esto es, con una elevada autonomía. Corresponderá a los agentes de socialización el establecimiento de metas que deberán orientar el comportamiento del socializado, que se producirá con una notable independencia respecto de normas rígidas y externamente impuestas. Mientras que en el modelo tradicional de socialización se enfatiza la necesidad de que los niños se adapten a los medios (los modos concretos de conducta) y a los fines (determinados valores sociales), el proceso de individualización o postmodernización ha propiciado que la crianza se conciba cada vez más orientada al segundo de los elementos citados. Ya Riesman (1981) se refirió al "carácter de dirección interna" en contraposición con el "carácter de dirección tradicional", como propio de las sociedades modernas. Sin embargo, al incorporar un tercer tipo, el "carácter de dirección por otros" pone de manifiesto los rasgos que identifican nuestras sociedades contemporáneas a las que etiqueta como sociedades de masas cuya emergencia tiene lugar a partir de mediados del siglo XX. En este modelo, se manifiestan importantes niveles de tensión, por sus objetivos contradictorios, entre los diferentes ámbitos socializadores: la familia, la escuela y la cultura del grupo de iguales, espoleada por los mass media. Así, el modelo de rígida autoridad familiar decae en un contexto de flexibilización de valores característico de las sociedades contemporáneas. Aparece una paradoja importante: a medida que es mayor el interés de los padres de procurar mayor nivel de autonomía a los hijos, habida cuenta de la necesidad de familiarizarlos pronto con los requerimientos de una sociedad cada vez más demandante de flexibilidad (Sennett, 2003), mayor es su grado de incertidumbre sobre las probabilidades de éxito de su tarea, dada la influencia de otros agentes socializadores.

La "institucionalización" tiene su origen en la expansión de estructuras organizativas en el contexto de racionalización de la vida social, tal como Weber apreció en su obra. Se trata del proceso a través del cual los niños se insertan en este tipo de estructuras siendo, en definitiva, destinatarios directos de su 
actividad. El sistema escolar es el ámbito en que más evidentemente se reconoce este proceso, aunque alcanza con especial intensidad a otras esferas (actividades de ocio, deporte, de tipo cultural o formativo...). La institucionalización, además, implica la "burocratización" en el comportamiento conforme a determinadas reglas para el logro de determinados fines o, en el sentido que menciona N. Elías (1987), constituye un factor fundamental en el proceso de civilización. La proliferación de los ámbitos burocratizados, de "jaulas de hierro", en el sentido weberiano (Weber), que restan oportunidades a la espontaneidad, creatividad y capacidad de encantamiento constituirían la cara negativa de este proceso creciente. Sin embargo, también se ha insistido en el interés de estos procesos como potenciadores de la interacción de iguales, dada la importancia central del criterio de edad a la hora de ordenar este tipo de organizaciones. Frente a la tendencia al control más próximo del escenario familiar, estos ámbitos permiten que, a pesar del marco más restrictivo, se desarrollen espacios informales de interacción a través de la cultura de iguales (Corsaro, 2003). Además, desde otros enfoques, se destacan otros aspectos positivos como que a través de estos ámbitos institucionales los niños se convierten en actores dignos de ser reconocidos específicamente como individuos en lugar de como una mera proyección de sus familias, o que se tienden a incrementar las capacidades comunicativas de los niños/as, dado el incremento de oportunidades de relación entre los iguales frente a las que se hacen posibles en el ámbito doméstico (Dencik, 1992).

Frones (1994) añade un tercer proceso, el de "individuación" como una dimensión eminentemente político-jurídica según la cual se ha producido una progresiva tendencia de los Estados modernos (y de los sistemas organizativos en general) a tratar a los individuos como unidades básicas en el marco de un proceso general de racionalización social que define a nuestras sociedades. Así, los derechos y deberes legales, incluyendo los de ciudadanía, son aplicados a los individuos y no a las familias. En el contexto de nuestras sociedades burocráticas, es ésta la mejor forma de garantizar no sólo un tratamiento igualitario entre los individuos, sino también un mayor grado de control sobre el cumplimiento eficiente de los objetivos que se pretenden. En relación con la infancia, "individuación" significa comprender a la familia no como una unidad inseparable, sino como conjunto de individuos depositarios de derechos que deberán ser garantizados con independencia de la interpretación que en su seno se realice de sus necesidades. La familia se convierte, así, en un instrumento fundamental para el cumplimiento de determinados requerimientos sociales que se establecen a través de las instituciones políticas y se 
garantizan, en última instancia, a través de instituciones de naturaleza administrativa y judicial ${ }^{1}$. Desde otra perspectiva, Donzelot (1979) sugiere que, con el surgimiento del Estado moderno, éste adquiere una serie de instrumentos para modelar a las familias. Quedan los criterios de intervención sobre éstas en manos de los aparatos del Estado, a través de normas dirigidas a retener y vigilar "adecuadamente" a los hijos bajo la amenaza de ser ellas mismas objeto de vigilancia y disciplinarización. Sea cual sea la opción teórica a través de la que se fundamente la "individuación", sí podemos reconocer en este concepto una dimensión relevante para la comprensión de la infancia moderna.

Hasta aquí, hemos analizado algunos aspectos definitorios de la concepción moderna de la infancia a partir de las dimensiones individualización, institucionalización e individuación. Tales aspectos nos remiten, como se ha visto, a factores estructurales en los que se concreta el tránsito hacia las sociedades contemporáneas. Sin pretender adentrarnos en el debate acerca de cómo las mutaciones estructurales afectan a los valores sociales, o viceversa, la cuestión es analizar qué concepciones respecto a la infancia (en particular, con relación a la crianza de los hijos) predominan en los modelos tradicional y contemporáneo, 0 , si se prefiere, materialista o postmaterialista. Es decir, si las condiciones estructurales se transforman en un proceso, resulta adecuado pensar que éste vendrá acompañado de otras transformaciones de índole cultural en lo relativo a los valores sociales. $\mathrm{Ni}$ que decir tiene que, de la misma forma en que las transformaciones sociales -en la mayoría de los casos- resultan de procesos paulatinos, acaso nunca del todo acabados, en el terreno de los valores ocurre algo parecido. Así, no se pasa del todo a la nada y, en nuestro objeto de estudio, los valores de tipo materialista en que los niños resultan meros proyectos de futuro que deben integrar todo un acervo de normas sociales preestablecidas, conviven con otros, de índole más contemporáneos, que se ajustan en mayor medida a concepciones de la infancia en las que se reclama un mayor grado de autonomía, en las que las relaciones se sustentan en presupuestos de confianza y en las que se confiere al individuo infantil una mayor capacidad participativa como individuo social, en definitiva, un mayor valor en sí mismo.

\footnotetext{
${ }^{1}$ Para profundizar sobre la cuestión de los derechos del niño: Archard, D. (2004). Children, Rights and childhood. Routledge. Londres.
} 


\section{VALORES SOCIALES Y LA PARTICIPACIÓN DE LA INFANCIA}

La cuestión siguiente nos conduce a analizar de qué modo y en qué medida la transformación social operada en las sociedades contemporáneas, y que tiene como resultado el desplazamiento hacia valores postmaterialistas ${ }^{2}$, ha implicado una transformación paralela en la relación adultos-infancia en el sentido de un mayor nivel de participación en la vida social por parte de los menores. O, dicho de otra manera, se trataría de averiguar si la transformación de los valores sociales (producto de la interacción de las dimensiones económica, cultural, política y de cualquier otro orden) ha resultado permeable a las relaciones con la infancia.

La apuesta por la participación de la infancia tiene una doble pretensión. Por un lado, la participación implica "responsabilización" en los propios asuntos y en aquéllos que les afectan de manera indirecta. Por otro, los cambios sociales que están operando en nuestra sociedad exigen que los niños desarrollen mecanismos que les permitan, como tales y, luego, como adultos, no ser simples sujetos pasivos de los mismos, sino controlar en lo posible su desarrollo, aprendiendo de esta forma a ser "flexibles, cooperativos, responsables y comprensivos"3. En definitiva, si, como resulta indiscutible, la infancia es un periodo tan crucial en la adquisición de pautas de socialización del sujeto, sólo en la medida en que los niños desarrollen un comportamiento participativo, pueden ver satisfechas sus necesidades como sujetos plenos en cuanto que niños, así como hacer posible su posición participativa como futuros adultos.

En el ámbito familiar, comprender este hecho supone transmitir valores que incidan en el reconocimiento del potencial interno del sujeto, que refuercen su autocontrol y que antepongan la independencia a la obediencia. Como en la literatura sociológica se menciona, estos valores se sitúan en el espectro "autonomíaheteronomía". En el primer caso se priorizan aquellos aspectos que inciden en el sometimiento del niño a normas que se imponen de forma ajena a los procesos de decisión interna del sujeto. El niño deberá aprender a comportarse conforme a unas

\footnotetext{
${ }^{2}$ Inglehart, R. Modernización y posmodernización : el cambio cultural, económico y político en 43 sociedades Publicación Madrid : Centro de Investigaciones Sociológicas. Siglo XXI, 2001

${ }^{3}$ Muñoz, C. (1995). La participación de la infancia y la juventud en la vida social. Promoció y participació de l'infancia i la juventud en la vida social. Ajuntament de Barcelona.
} 
pautas establecidas externamente por los sujetos socializadores (de acuerdo a un modelo "cartesiano", en el que el proceso educativo del niño está marcado por normas que tienden a resultar rígidas). En el segundo, en cambio, lo relevante estará más en las potencialidades inherentes del niño, en el descubrimiento de su genio interior (como se expresa en la pedagogía "roussoniana") (Gil Calvo, 2003).

No obstante, no parece que la transformación de los valores de la que ha dado cuenta nuestra sociedad haya sido tan aguda en el terreno de la relación con la infancia. F. Casas (1998) se refiere a que en el núcleo figurativo de las representaciones en torno a la infancia, es decir, en lo que constituye los aspectos más esquivos al cambio, se encuentra la idea de "aún no". Qvortrup (1992) afirma que la realidad del niño como actor y contribuyente a la sociedad ha sido eliminada en gran parte en la sociedad moderna. Sin embargo, Jensen \& MacKee (2003) afirman que determinados cambios estructurales han llevado a que las relaciones entre padres y niños se produzca en términos más democráticos, por la asunción de un mayor poder para la negociación por parte de éstos. En términos parecidos, Flaquer (1998) alude a la preferencia por las relaciones más flexibles e íntimas entre padres e hijos, y Alberdi (1999) a la tendencia a la "simetría familiar", que no sólo alcanzará a los vínculos de pareja, sino también a las relaciones de los padres con los hijos.

En cualquier caso, podríamos plantearnos la hipótesis de que existe un desfase en la transformación de los valores sociales hacia pautas postmaterialistas y la asunción de este tipo de valores con relación a la infancia, dada la persistencia de las concepciones más tradicionales respecto del papel de los niños en nuestra sociedad. Matizaríamos esta hipótesis en el siguiente sentido: a medida que se asumen relaciones de tipo familiar con niños, aumenta la probabilidad de que las concepciones más tradicionales se "actualicen" hacia niveles más participativos. Corresponde a continuación analizar este hecho, para lo cual compararemos la población que se muestra favorable a valores materialistas y postmaterialistas, analizando la medida en que ambos grupos tienden a adoptar pautas de socialización "heterónomas" o "autónomas" respectivamente. 


\section{LOS VALORES DE LOS ADULTOS RESPECTO A LA INFANCIA: EL DILEMA AUTONOMÍA-HETERONOMÍA}

Para el estudio empírico de esta cuestión, nos serviremos de la base de datos de la Encuesta Mundial de Valores, en lo relativo a los datos recabados para Andalucía en el año 1998. De esta encuesta, que por su naturaleza trata de abordar el análisis sobre valores sociales con relación a cuestiones muy diversas, hemos seleccionado aquéllos ítems especialmente útiles para el objetivo de esta investigación, esto es, aquellos que nos informan sobre las concepciones de los adultos respecto a la infancia en el contexto de las relaciones familiares. Concretamente la P8 pregunta a partir de "una lista de cualidades que se pueden inculcar a los hijos en el hogar, ¿cuál considera $\mathrm{Vd}$. especialmente importante?" Las opciones de respuesta, sobre las que se solicita mencionar un máximo de cinco, son buenos modales (V14), independencia (V15), esfuerzo en el trabajo (V16), sentido de la responsabilidad (V17), imaginación (V18), tolerancia y respeto hacia los demás (V19), sobriedad y espíritu de ahorro (V20), determinación, perseverancia (V21), fe religiosa (V22), abnegación (espíritu de sacrificio) (V23), obediencia (V24). Se propone aquí un listado de cualidades (aunque como veremos, no todas) que se vinculan a un estilo de socialización alternativamente "autónomo" o "heterónomo", resultando el primero más cercano a valores postmaterialistas y el segundo más cercano a un modelo materialista, en los términos teóricos manejados por Inglehart (1998).

La preponderancia de las pautas "heterónomas" nos dibuja un panorama social más próximo al estadio tradicional (si se prefiere, materialista), en que los hijos tienen importancia en su dimensión "proyecto", en el que la transmisión de normas y la adecuada recepción de las mismas es el objeto del proceso socializador y en el que se valora el sacrificio sobre el esfuerzo, la fe sobre la imaginación y la obediencia sobre la independencia. El individuo considera oportuno transmitir valores más próximos a modelos autoritarios que democráticos: la participación en la toma de decisiones familiares se cierra paso en favor de los comportamientos predeterminados por la tradición o la autoridad del progenitor. 
Cuadro 1. Cualidades que inculcaría a los hijos en el hogar. Encuesta Mundial de Valores 1996

\begin{tabular}{|l|c|c|c|c|c|c|}
\hline & $\begin{array}{c}\text { Buenas } \\
\text { maneras }\end{array}$ & Independencia & $\begin{array}{c}\text { Esfuerzo } \\
\text { trabajo }\end{array}$ & Responsabilidad & Imaginación & Tolerancia \\
\hline Mencionado & $86.9 \%$ & $22.4 \%$ & $63.7 \%$ & $77.2 \%$ & $17.5 \%$ & $78.6 \%$ \\
\hline No mencionado & $13.1 \%$ & $77.6 \%$ & $36.3 \%$ & $22.8 \%$ & $82.5 \%$ & $21.4 \%$ \\
\hline & $\begin{array}{c}\text { Espíritu } \\
\text { ahorro }\end{array}$ & Determinación & Religión & Abnegación & Obediencia & \\
\hline Mencionado & $14.4 \%$ & $13.8 \%$ & $27.2 \%$ & $10.2 \%$ & $51.1 \%$ & \\
\hline No mencionado & $85.6 \%$ & $86.2 \%$ & $72.8 \%$ & $89.8 \%$ & $48.9 \%$ & \\
\hline
\end{tabular}

En términos generales, la sociedad andaluza presenta un mayor grado de identificación con cualidades de tipo heterónomas, esto es, de carácter materialista, en comparación con el resto del país. De todas las opciones propuestas, las "buenas maneras" resultan ser la cualidad más mencionada por los encuestados como susceptible de inculcar a sus hijos: el 86,9\% mencionó como relevante este aspecto (en total se pudo elegir hasta cinco cualidades). De la relación propuesta de cualidades, el segundo factor en importancia resultó ser la "tolerancia", al que descartamos del dilema autonomía-heteronomía, por cuanto parece evidente que aquélla no se relaciona con la preferencia en valores que insisten en el control interno o externo sobre el individuo: el 78,5\% menciona la tolerancia como un aspecto fundamental para inculcar a sus hijos (tres puntos por encima de la media del Estado). Seguidamente, la cualidad de la "responsabilidad", que finalmente hemos descartado de identificar como valor autónomo (por su ambigüedad, al poderse entender tanto como la obligación del individuo de responder de los propios actos como la orientación del sujeto a responder ante los incumplimientos de normas externas), ha sido mencionada por un porcentaje del $77,3 \%$.

Sólo superó el 50\%, además de las opciones citadas, un valor típicamente heterónomo como es la "obediencia" (concretamente fue mencionada por el 51\% de los casos). Debemos resaltar que las opciones menos secundadas por los encuestados corresponden a dos cualidades que ejemplifican los dos extremos del continuum autonomía-heteronomía: sólo el 10\% mencionó la "abnegación" (valor que incluimos como valor "autónomo" por lo que implica de renuncia de la individualidad para un fin determinado, externamente inducido). Tan sólo el 13\% optó por la "determinación" (que igualmente incluimos dentro del campo semántico de la "heterónoma" por cuanto la consideramos como una cualidad que invita a una situación extrema del autocontrol, esto es, capacidad para imponer las propias 
decisiones). Obtienen valores intermedios la "religión" (27,4\%), la "independencia" (22.5\%) y la "imaginación" (17\%).

\begin{tabular}{|c|c|c|c|}
\hline \multirow{5}{*}{ Valores Materialistas } & Heterónomos & Autónomos & \multirow{5}{*}{ Valores postmaterialistas } \\
\hline & Buenas maneras & Independencia & \\
\hline & Obediencia & Imaginación & \\
\hline & Fe religiosa & Determinación & \\
\hline & Abnegación & Esfuerzo en el trabajo & \\
\hline
\end{tabular}

Resulta, así, evidente qué cualidades destacan los padres como aquéllas que consideran importante inculcar a sus hijos y, de igual forma, cuáles no. A continuación, realizaremos una doble labor de análisis, profundizando, en primer lugar, en la dicotomía heteronomía-autonomía, para lo que construiremos un indicador sintético que nos permitirá definir en qué posición se sitúan los individuos de la muestra respecto a esta alternativa, incluyendo el valor "mixto" para aquéllos que registren valores altos en las dos opciones ${ }^{4}$. En segundo lugar, también hemos optado por profundizar en el análisis de alguna de las cualidades que han sido presentadas con anterioridad, deteniéndonos, por su relación específica con la cuestión que nos ocupa, en la cualidad "obediencia", y ello a través del análisis de relaciones estadísticas con diferentes variables tomadas como independientes.

Para obtener este índice, hemos considerado heterónomos a aquellos sujetos muestrales que mencionan al menos dos cualidades heterónomas de las cuatro propuestas (buenas maneras, fe religiosa, obediencia, abnegación), siempre que no hayan mencionado más de una cualidad autónoma, y lo mismo para definir a los sujetos "autónomos" (habiéndose propuesto las cualidades independencia, determinación, imaginación, esfuerzo en el trabajo), considerando mixtos al resto. Los valores perdidos corresponden a aquéllos que no satisfacen ninguna de las condiciones mencionadas. Hay que considerar que la categoría autonomíaheteronomía se ha construido dando al encuestado la opción de mencionar cinco entre diez cualidades que se les ofrecen, de las cuales cuatro han sido descartadas por ser escasamente relevantes para la medición del índice. En cualquier caso, a

\footnotetext{
${ }^{4}$ En cualquier caso, para esta cuestión, nos remitimos también al análisis que realizan Del Pino y Bericat basándose en las dimensiones citadas, a partir de las variables sociodemográficas que se han indicado, aunque utilizando un procedimiento de análisis multivariable diferente del que aquí se propone. Del Pino, J et. Bericat, E. (1998). Op. Cit. Pág. 52-59.
} 
través de este índice obtenemos información consistente en cuanto que nos permite saber cuántos individuos han optado preferentemente por cualidades de una u otra dimensión.

\begin{tabular}{|l|l|c|c|c|c|}
\hline \multicolumn{5}{c}{ Cuadro 2. Preferencia por valores autónomos o heterónomos } \\
\hline & & Frecuencia & Porcentaje & Porcentaje válido & $\begin{array}{c}\text { Porcentaje } \\
\text { acumulado }\end{array}$ \\
\hline \multirow{2}{*}{ Válidos } & Heterónomos & 995 & 55.2 & & \\
& Autónomos & 550 & 30.5 & 58,9 & 58,9 \\
& Mixtos & 144 & 8.0 & 32,6 & 91,5 \\
\hline \multirow{3}{*}{ Perdidos } & Total & 1.689 & 93.7 & 8,5 & 100,0 \\
\hline Total & Sistema & 114 & 6,3 & 100,0 & \\
\hline
\end{tabular}

Analizando sus resultados, observamos que la opción "heterónomos" (58,9\%) obtiene puntuaciones superiores al valor "autónomos" (32,6\%), alcanzando la categoría "mixtos" un porcentaje del $8,5 \%$ (si se toman como referencia los porcentajes válidos). Por ello confirmamos la tendencia sobre la vinculación de la sociedad andaluza con valores de tipo heterónomos. Sin embargo, consideramos oportuno profundizar en cómo el posicionamiento en una u otra opción está condicionado por diferentes variables que se toman como independientes.

Cuadro 3. Resultado del análisis de significatividad entre variables tipo de orientación a la socialización (heterónoma/autónoma) y diversas variables independientes

\begin{tabular}{|l|c|c|c|c|c|}
\hline & Import. familia & Mater./Postmat. & Clase Social & Formac. & NoHijos \\
\hline \begin{tabular}{l|c|c|c|c|}
\hline Heterónomos \\
Autónomos
\end{tabular} & + & + & + & + & + \\
\hline
\end{tabular}

\begin{tabular}{|l|c|c|c|c|}
\hline & Sexo & Edad & Status Marital & $\begin{array}{c}\text { Tamaño } \\
\text { Poblac. }\end{array}$ \\
\hline $\begin{array}{l}\text { Heterónomos/ } \\
\text { Autónomos }\end{array}$ & + & + & + & - \\
\hline
\end{tabular}

$$
\begin{aligned}
& (+: \text { relación significativa, }<0.05 \text { en coeficiente de Chi-cuadrado) } \\
& (-: \text { relación no significativa, }>0.05 \text { en coeficiente de Chi-cuadrado }
\end{aligned}
$$

Estos resultados son perfectamente predecibles en el sentido de que la preferencia por cualidades heterónomas 0 autónomas tienden a condicionarse a la adscripción a valores materialistas o postmaterialistas que correlacionan, a la vez, con variables sociodemográficas como las mencionadas arriba. Así, las cualidades heterónomas se valoran más entre las clases trabajadora y baja, en los niveles más precarios de formación, entre las mujeres, en las edades más avanzadas, entre los 
sujetos casados o viudos, y entre aquellos sujetos con mayor número de hijos. En sentido contrario, predominan las cualidades autónomas en las clases medias (no así en la alta), entre los sujetos más formados, entre los hombres, en los más jóvenes, en los separados y divorciados, y en los individuos sin hijos.

\section{LA OBEDIENCIA Y LA INDEPENDENCIA COMO CUALIDADES MODELO. ANÁLISIS DE LAS RELACIONES ENTRE ESTAS CUALIDADES Y VARIABLES DEPENDIENTES}

Como apuntábamos más arriba, si tomamos como referencia las cualidades por separado, los ciudadanos andaluces están más interesados en transmitir "buenos modales", "esfuerzo por el trabajo" que cualidades como la "imaginación", la "determinación" o la "abnegación". No obstante, mantienen un fuerte apego a cualidades típicamente "heterónomas" como la "obediencia", que nos remite directamente al modo en el que se desenvuelven las relaciones paterno-filiales. No dudamos de que la obediencia deba interpretarse como un valor aplicable a las relaciones sociales en el sentido más amplio, fundamentadas, como es bien sabido, en el principio de jerarquía. Especialmente en las clases trabajadoras, saber obedecer puede entenderse como un valor cuyo aprendizaje durante la infancia pueda considerarse adecuado para el desenvolvimiento del individuo una vez se alcance la edad madura. Sea por un motivo de utilidad, sea por otro diferente, en este caso reconoceríamos la "obediencia" como "valor en sí mismo", si se prefiere, esencial. Podría entenderse, sin embargo, que este valor tienda a ser residual en la medida en que las sociedades formalmente se democratizan y la iconografía clasista, a partir del espejismo de la sociedad de consumo, se desmorona. En la medida en que aumentan las posibilidades de ascenso social, o así se perciba, la transmisión del valor de la obediencia debería perder peso en favor de otros como la determinación, que responde a su antónimo semántico (capacidad de actuar con autonomía).

Sin embargo, el valor de la obediencia continuaría siendo importante en la medida en que se juzgue esencial para transformar a los niños de "potenciales" (becoming being) en seres humanos propiamente dichos (human being): es decir, si se juzga en cuanto que instrumento de socialización antes que como valor a socializar. Se podría incluso admitir que aprender a obedecer es cada vez menos necesario en nuestras sociedades, siendo más funcional la flexibilidad, la capacidad 
para las relaciones, para el aprendizaje... Sin embargo, podemos sospechar que este valor continúa teniendo vigencia en la relación con los propios niños, es decir, cuando este rol lo desempeña el sujeto infantil dependiente. En este caso, identificamos el valor "obediencia" como valor instrumental. Como decíamos, planteamos la hipótesis de que en la medida en que en las sociedades contemporáneas ceden terreno los valores autoritarios, como la obediencia, en las relaciones paterno-filiales cuentan con un alto nivel de resistencia, denotando este hecho que el cambio general de valores que enfrenta la postmaterialización social afecta en menor medida a los niños, que se perpetuarían así como actores con un papel meramente dependientes, en función de los intereses del mundo adulto.

Pretendíamos demostrar este hecho, para lo cual nos servimos de diferentes cruces de variables a través de tablas de contingencia, entre la variable V24 -obediencia- y otras escogidas como independientes, detectando las relaciones estadísticamente significativas (Sig. asintótica (bilateral)<0.05): "materialista/ postmaterialista", "clase social", "educación", "edad", "estatus marital" y "tamaño de la población" y "número de hijos". Partíamos de que la "obediencia" tiene una doble dimensión de valor instrumental en la relación con los hijos (especialmente con los niños) y valor esencial, en cuanto que se entiende valor en sí mismo. Si bien el resto de las relaciones se entienden por la mayor presencia de valores postmaterialistas en las clases medias, los más formados, los más jóvenes, y de ámbitos urbanos, la relación "status marital"/"obediencia", resultaba interesante comprobar la existencia de significatividad en la relación estadística cuando se vinculaba la variable "obediencia" con la variable "status marital" (positiva con valores de casado/a, en pareja de hecho, divorciado/a, separado/a, viudo/a, soltero/a), siendo los individuos casados $(51,2 \%)$, aquéllos que viven como pareja de hecho (50\%) y los viudos/as $(65,3 \%)$ los que mencionan en mayor porcentaje esta cualidad (frente a solteros $(47,8 \%)$, separados $(45,5 \%)$ y divorciados $(41,7 \%))$.

Sin embargo, lo fundamental aquí es analizar si la "obediencia" se concebiría como un valor con una notable carga "instrumental", en el sentido de que serían aquéllos que tienen mayores probabilidades de convivir con niños los que más enfatizarían esa cualidad. 


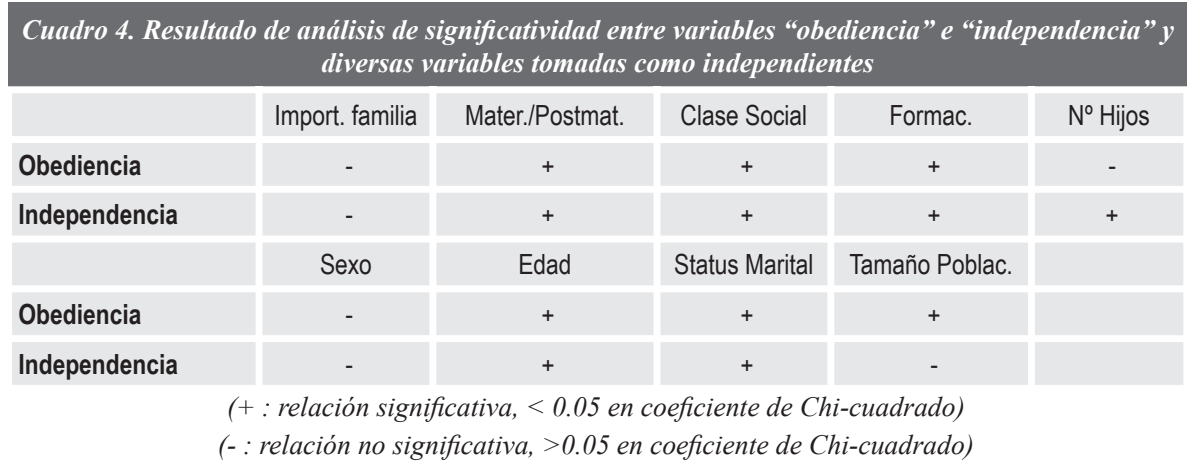

Al tomar como referencia la variable "número de hijos" (que hemos recodificado para facilitar el análisis), observamos cómo resulta complicado sostener esta hipótesis ya que el mayor énfasis en el valor "obediencia" no se vincula al hecho de tener o no tener hijos, ni siquiera al hecho de tener mayor o menor número de hijos. Por tanto, el énfasis en inculcar la cualidad de la obediencia trasciende la realidad fáctica de la relación con los propios hijos sino que, podríamos pensar, se vincula al universo valorativo de los sujetos en términos más o menos materialistas o postmaterialistas. No por el hecho de que los individuos tengan hijos, o incluso muchos hijos experimentarán mayor interés por garantizar su obediencia. La "obediencia", así, se entiende como un valor que tiende a restringirse a aquellos sujetos cuyo perfil de clase, de formación, de hábitat, etc., les sitúan en una perspectiva materialista sobre la realidad social.

Como decíamos, la variable "obediencia" tiene una relación estadística significativa con la "clase social subjetiva", partiendo de la hipótesis teórica según la cual la obediencia, como cualidad heterónoma más reconocible, se configura como un valor más apreciado en las clases trabajadora y baja que en la clase media (Kohn, 2001). Este hecho, repetidamente analizado en la literatura sociológica, se explicaría por la mayor tendencia de los trabajadores manuales a inculcar valores de tipo heterónomo, en contraste con lo que ocurre con los trabajadores de "cuello blanco". Según los resultados de esta encuesta, mientras que el $53 \%$ y $64,9 \%$ de las clases trabajadora y baja, respectivamente, mencionaron entre las facultades relevantes la "obediencia", sólo lo hicieron el $48,7 \%$ y $39,2 \%$ de la clase media-baja y media-alta, respectivamente. 


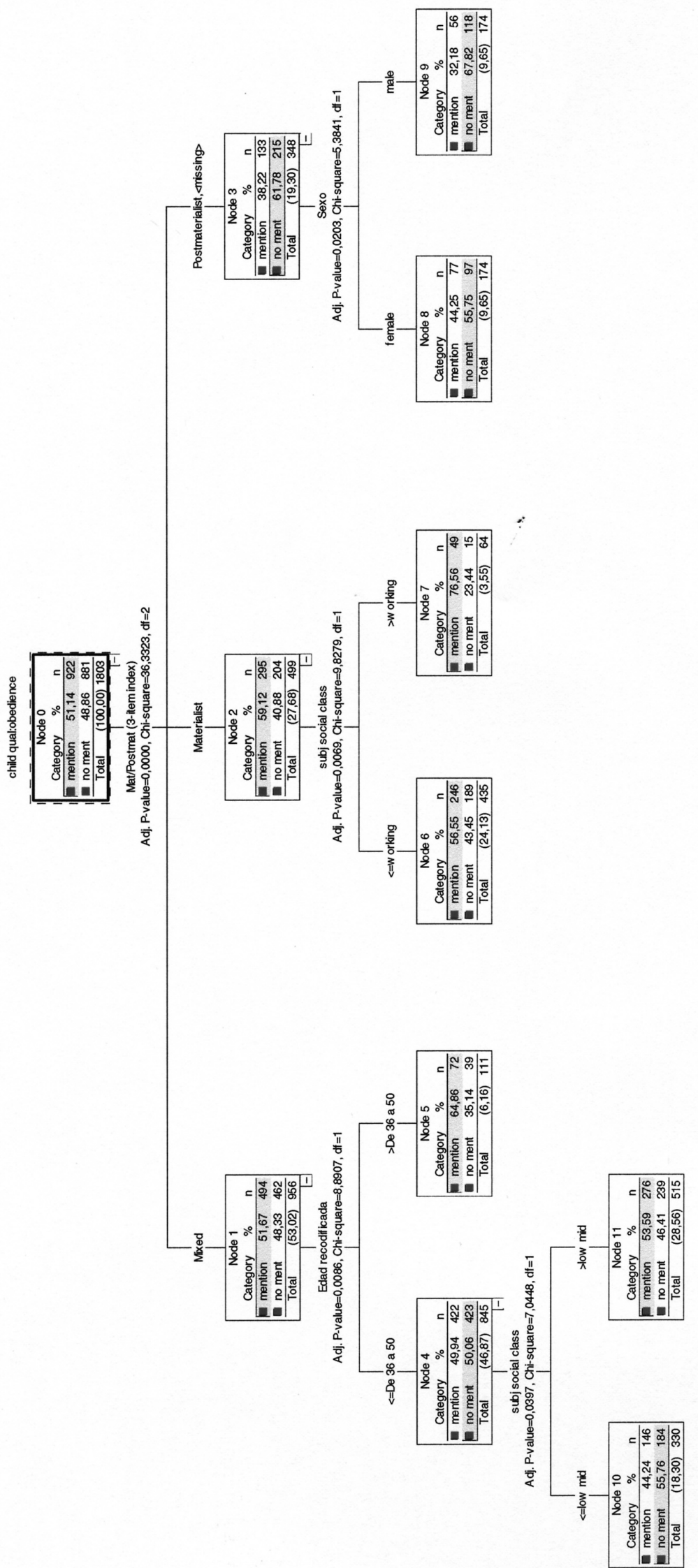


Complementando lo reseñado, la relación significativa (sig. Chi-cuadrado de Pearson= 0.000) entre la variable "obediencia" (repetimos, como cualidad que se considera oportuno inculcar a los hijos) y la variable sintética que agrupa a los individuos materialistas, postmaterialistas o mixtos, confirma la obediencia como cualidad que se valora "en sí misma" antes que como instrumento más eficaz de socialización. A tenor de lo expuesto, no parece en absoluto sorprendente este hallazgo, dado que los individuos de clase media tienden a presentar estilos de vida típicamente postmaterialistas, al contrario de lo que ocurre con individuos de clase trabajadora, más proclives a asumir valores materialistas. Este hecho se explica de modo sencillo: un mayor nivel de satisfacción de las necesidades de supervivencia lleva a dar más importancia a metas postmaterialistas, lo cual explica que en la clase media-alta se dé un porcentaje de individuos materialistas del $19,5 \%$ y en la clase baja éste ascienda hasta el $56,1 \%{ }^{5}$. No obstante, en consonancia con lo que indicábamos, otros factores pueden ayudar a explicar este hecho, como que un mayor nivel de formación permite mayor grado de familiaridad con las exigencias de abstracción que demanda un modelo de socialización en clave más participativa (Kohn, 1996).

Tomando como variable dependiente la "independencia", como cualidad autónoma más valorada, encontramos que las relaciones no difieren mucho respecto de la que enunciamos respecto de la cualidad "obediencia". Es más, tan sólo en lo que afecta al tamaño de la ciudad y al número de hijos, la relación difiere de la que se evidencia en la otra cualidad analizada. Quizás merezca una adicional explicación por qué se pude producir esta diferencia entre los resultados obtenidos con relación a la cualidad "obediencia" y éstos que se refieren a la cualidad "independencia", cuando podríamos a priori intuir que la relación entre una y otra es de oposición semántica.

En primer lugar conviene apuntar que el número de hijos afecta a la opinión sobre el valor de la independencia de la siguiente forma. Como es previsible, a medida que se incrementa el número de hijos, desciende el interés por esta cualidad (lo cual ocurre hasta el número de 7 hijos, momento en el cual el número de casos, como es lógico, ya es muy reducido). De ello se deduce que, así como la obediencia no se condiciona al número de hijos, el que hemos entendido como su valor antagónico -la "independencia"- sí lo hace de una forma previsible. Así como la obediencia

\footnotetext{
${ }^{5}$ No obstante, la mayoría de los sujetos agregados en clases subjetiva obtienen valores mixtos, siendo éste el valor que registra la moda en todos y cada uno de los agregados de clase.
} 
decae como valor esencial de la relación entre progenitores e hijos, dada su escasa funcionalidad en un contexto de profundo cambio, con el valor de la independencia ocurre lo contrario y se eleva como valor en alza, especialmente entre quienes tienen menor número de hijos (el $23,7 \%$ de los que tienen un hijo, el $24,6 \%$ de los que tienen dos y sólo el de $16,8 \%$ de los que tienen tres). Para los primeros es relativamente más importante que los hijos sean independientes, lo cual parece que a priori tiene que ver con otras variables implicadas. La cuestión es conocer qué variables pueden estar afectadas en la explicación de este hecho y en qué orden. Indagar en el perfil social de quienes más propenden a elegir una cualidad u otra nos ayudaría a resolver esta incógnita.

Para realizar esta labor nos ayudamos del análisis de segmentación. A través de este instrumento, después de seleccionar una serie de variables de acuerdo con las hipótesis generadas en la investigación, se produce un proceso de segmentaciones sucesivas que nos permitirá mostrar la influencia de las diferentes variables a través de la forma gráfica de un árbol (el software que realiza esta tarea se denomina Answer tree). La ordenación de las variables se produce según el grado de predicción de cada una de éstas en relación con la variable dependiente. Así, el primer nivel estará ocupado por la variable que mejor prediga los resultados obtenidos en aquélla y así sucesivamente. En nuestro caso, hemos introducido las variables independientes mencionadas con anterioridad a las que se aplicó un análisis "chi-cuadrado". Como resultado de la aplicación del procedimiento de segmentación, hemos obtenido los siguientes niveles.

La variable que actúa con mayor potencial predictor resultó ser la relativa a la orientación respecto del dilema materialista-postmaterialista, obteniendo los resultados más elevados respecto de la mención de la cualidad "obediencia" $(59,12 \%)$ aquéllos que se sitúan en posiciones materialistas. Apenas superan la mitad de ese porcentaje quienes se sitúan en el postmaterialismo. Esto resultaba del todo previsible, por cuanto que los valores heterónomos se asocian con nitidez con el universo significativo materialista, al contrario de lo que ocurre con los valores autónomos. Sin embargo, merece una explicación adicional el hecho de que en el caso de quienes se vinculan a valores materialistas, los que se autoposicionan en la clase media o superior tienden a mostrar actitudes más favorables a la obediencia, en un porcentaje, además, extraordinariamente elevado (76,56\%). Se intuye cierta polarización entre quienes se sitúan en la clase media o superiores y manifiestan 


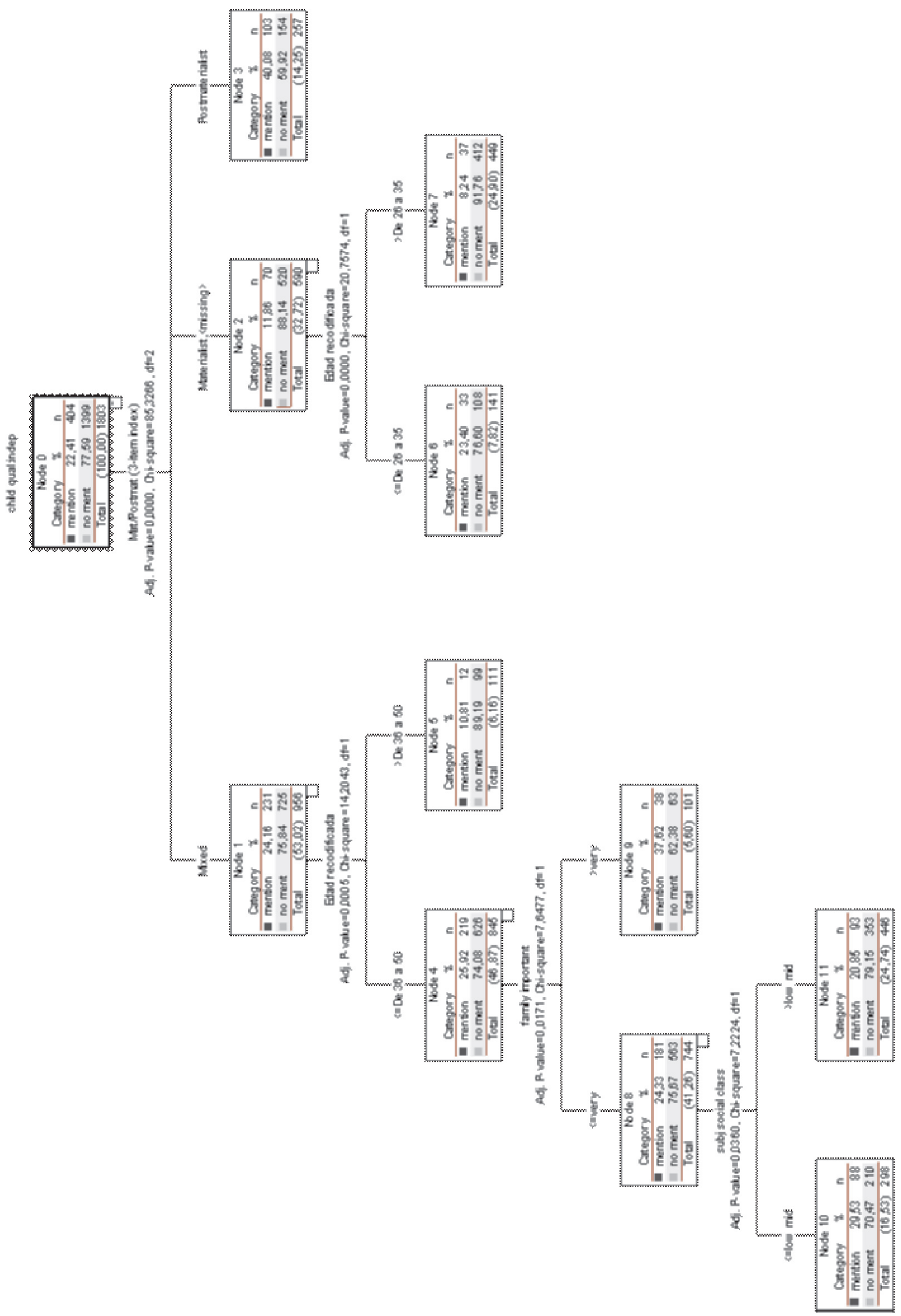


crianza y aquéllos que se posicionan a favor de la otra alternativa. Recordemos que la relación entre clase social y orientación a la obediencia es significativa y negativa, es decir, que a medida que se incrementa en el nivel de clase, disminuye la propensión a aquel valor. Algo parecido ocurre con quienes se adhieren a valores mixtos (del dilema materialismo-postmaterialismo) dentro del grupo de edad comprendido entre los $18 \mathrm{y}$ 36 , en el que se muestra mayor propensión a mencionar esta cualidad entre quienes se sitúan en clases medias altas y superiores (53,59\%). De igual manera, entre las mujeres "postmaterialistas" se incrementa la valoración por la cualidad "obediencia", lo cual parece corresponder con la mayor vinculación al hecho socializador que sistemáticamente se comprueba en las mujeres, dado los roles que se les atribuye predominantemente, en el que la obediencia se puede percibir como una condición necesaria para que ese hecho socializador se produzca adecuadamente.

La "independencia" es mencionada principalmente por quienes se muestran partidarios de valores de tipo postmaterialista (en un $40,08 \%$ ) frente a los que se pronuncian a favor de valores materialistas $(11,86 \%)$ o mixtos $(24,16 \%)$. La edad es la otra variable influyente en un segundo escalón, resultando que los más jóvenes puntúan más alto en esta cualidad tanto entre los que se inclinan por valores materialistas como entre los que se inclinan por valores mixtos. Es destacable que son más proclives a esta cualidad los que, no superando los 50 años, tienen valores mixtos en el dilema materialismo-postmaterialismo y reconocen que la familia es una institución muy importante.

Como se observa en este análisis, el universo valorativo del sujeto, en términos de mayor o menor nivel de proximidad a valores modernos y postmodernos, es el factor más influyente de cuantos se han tenido en cuenta a la hora de explicar la proximidad a pautas de crianza más o menos autónomas. La edad, especialmente en cuanto a la "independencia" y, junto con la edad, la clase social y el sexo en cuanto a la "obediencia", constituyen el segundo nivel de influencia estadística a la hora de explicar la proximidad a uno u otro tipo de cualidades. Por tanto, la situación fáctica respecto a la condición de padres o madres no afecta significativamente a la preferencia por estas cualidades. En el caso de la "obediencia", ni siquiera la relación es significativa entre ésta y el número de hijos (o tenerlos o no tenerlos). En el caso de la "independencia", aunque sí existe relación significativa, ésta parece ser espuria frente a otras que adquieren mayor grado de validez, por lo que el número de hijos puede ser antes consecuencia de una determinada combinación de variables - entre 
las que adquiere especial significación el universo valorativo del individuo- que causa de determinadas preferencias en la crianza de éstos.

\section{CONCLUSIONES}

Por tanto, de los datos ofrecidos por la Encuesta Mundial de Valores para nuestra Comunidad Autónoma, no se deduce que exista algún modo de desfase entre los valores que la sociedad andaluza proyecta en general, en términos materialistas y postmaterialistas, con respecto a las representaciones predominantes en su universo valorativo sobre la infancia. Observamos cómo las transformaciones sociales operadas en nuestra sociedad son importantes dinamizadores del cambio en la concepción valorativa de los ciudadanos, registrándose, no obstante, mayor tendencia a la identificación de los andaluces con valores heterónomos. Se puede esperar que, a medida en que se profundice en la transformación estructural de nuestra sociedad hacia perfiles más asimilables con las realidades de nuestro entorno, los valores postmaterialistas registrarán una mayor expansión que arrastrará a las concepciones predominantes sobre los niños hacia claves de mayor reconocimiento de su "status" social, de su participación como individuos plenamente sociales.

Pero, al mismo tiempo, no creemos que el fomento de pautas participativas dependa en exclusiva del orden de valores predominantes en una sociedad. Es necesario huir de cualquier forma de determinismo estéril que no reconozca la interacción de diversos factores en el desenvolvimiento de las sociedades. En este sentido, existen fuerzas contrapuestas que presionan al reconocer mayor 0 menor peso a la participación social de la infancia. A través de este artículo, hemos considerado la conveniencia de analizar en qué medida los valores de los adultos se encuentran inclinados a aceptar modelos de relación más participativa con la infancia. Hemos observado que los valores sociales respecto del papel que debe representar el mundo infantil encuentran un directo correlato con el resto de las concepciones sobre la realidad social en general. 


\section{BIBLIOGRAFÍA}

CANO GARCÍA, G. y GARCÍA GÓMEZ, A. (2000). "Sistema de ciudades andaluzas". En Cano, G. (Dir.) Conocer Andalucía. Gran Enciclopedia Andaluza del Siglo XXI. Vol. 5: Infraestructuras y Ordenación del Territorio. Sevilla, Ediciones Tartessos, pp. 11-71.

Aguinaga, J y Comas, D. (1991). Infancia y Adolescencia: la mirada de los adultos. Ministerio de Asuntos Sociales. Madrid.

Alberdi, I. (1999). La nueva familia española. Publicación Madrid. Taurus.

Beck, U. (2002). Individualization. Institucionalized Individualism and its social and political consequences. Sage Publications. London.

Brannen, J. y O'Brien, M. (Ed.) (1996). Children in families : research and policy. Falmer Press. London; Washington, D.C.

Casas, F. (1998). Infancia: perspectivas psicosociales. Paidós. Barcelona.

Corsaro, W.A. (1997). The sociology of childhood. Thousand Oaks, California : Pine Forge.

Corsaro, W. (2003). "We're Friends, Right?”: Inside Kids' Cultur.: Joseph Henry Press. Washington D.C.

Del Pino, J. y Bericat, E. (1998). Valores sociales de la cultura andaluza. Encuesta Mundial de Valores. Andalucía 1996. Centro de Investigaciones Sociológicas. Madrid.

Dencik, L. (1992). "Creciendo en la era postmoderna: el niño y la familia en el Estado del Bienestar". En Anuario de Psicología, $n^{0} 53$. Universitat de Barcelona.

Donzelot, J. (1979). La policía de familias. Pre-textos. Valencia. Citado en IOE (1989), "Infancia moderna y desigualdad social". En Documentación Social. № 74. EneroMarzo 1989. 
Elías, N. (1987). El proceso de la civilización: investigaciones sociogenéticas y psicogenéticas. Publicación Madrid [etc.] Fondo de Cultura Económica.

Flaquer, LI. (1998). El destino de la familia. Barcelona: Ariel.

Frones, I. (1994). "Dimensions of Childhood". En Qvortrup, J, Bardy, M, Sgritta, G,

Weber, M. (2002). Economía y sociedad. Edición preparada por Johannes Winckelmann ; nota preliminar de José Medina Echavarría. Fondo de Cultura Económica. México, D.F.

Wintersberger, H. (Eds.). Childhood Matters. Social Theory, Practice and Politics. European Centre of Viena. Avebury.

Gaitán, L. (1999). El espacio social de la infancia. Comunidad de Madrid. Consejería de Sanidad y Servicios Sociales,

Ghiolla, M.N. (1994). "Day Care. Adult Interests versus Children's Need? A question of Compatibility". En Qvortrup, J, Bardy, M, Sgritta, G, Wintersberger, H. (Eds.). Childhood Matters. Social Theory, Practice and Politics. European Centre of Viena. Avebury

Gil Calvo, E. (2001). Nacidos para cambiar: cómo construimos nuestras biografías. Taurus. Madrid.

Inglehart, R. (1998). Modernización y Postmodernización. El cambio cultural, económico y político en 43 sociedades. Centro de Investigaciones Sociológicas. Madrid.

Jensen, A. y McKee, L. (2003). Children and the changing family.

Betweeen transformation and negotiation. London. RoutledgeFalmer.

Kohn, M.L. (1999). "Relaciones paterno-filiales y clase social". En Fernández-Enguita, M. (Ed.). Sociología de la Educación. Ariel Referencia. Barcelona.

Muñoz, C. (1995). La participación de la infancia y la juventud en la vida social. Promoció y participació de l'infancia i la juventud en la vida social. Ajuntament de Barcelona. 
Prout, A. "Children's Participation: Control and Self-realisation in British Late Modernity". En Children and Society, 14, 4. 304-15.

Qvortrup, J (1992). "El niño como sujeto y objeto: ideas sobre el programa de infancia en el Centro Europeo de Viena". En Infancia y Sociedad. № 15. Ministerio de Asuntos Sociales.

Qvortrup, J (1993). "Nine theses about "Childhood as a Social Phenomenon" en Qvortrup, J. Childhood as a Social Phenomenon. Lessons from an International Project, Viena. European Centre for Social Welfare Policy and Research.

Saporiti, A. (1994) "A Metolodogy for Making Children Count". En Qvortrup, J. y al. (eds.) Childhood matters. Avebury. Adershot.

Sennett, R. (2003). La corrosión del carácter : las consecuencias personales del trabajo en el nuevo capitalismo. Traducción de Daniel Najmías. Anagrama. Barcelona. 